\title{
New Insights on Diagnosis and Treatment of Cerebral Venous Thrombosis - A Narrative Review
}

\author{
Thomas Gabriel Schreiner
}

\section{ABSTRACT}

Cerebral venous thrombosis (CVT), although accounting for only $0.5-1 \%$ of all strokes, remains a potentially fatal neurological emergency, which must be promptly diagnosed and treated. Consisting of two parts, this review aims to highlight the most important data from recent years regarding diagnosis and treatment of CVT, illustrating both the current modern therapeutic approach and the future research directions in the field. Regarding the clinical diagnosis, the neurologist may sometimes have difficulties in recognizing this pathology, given the diverse clinical picture of the acute stage of CVT that can mimic various neurological disorders. In addition, although most risk factors (procoagulation status, infections, trauma, systemic diseases) are known and can be easily detected, in a significant percentage of cases the etiology remains uncertain. For paraclinical diagnosis, among the imaging investigations essential to support the diagnosis, CT angiography and MRI venography are reliable alternatives to digital subtraction angiography which represent the gold standard nowadays. In terms of treatment, international guidelines provide general directions for anticoagulation, with low molecular weight heparin being highly recommended. Regarding invasive treatment methods (thrombolysis, thrombectomy) that could be used in severe cases where anticoagulation has been shown to be insufficient, as well as symptomatic therapy, the evidence is often insufficient, new randomized clinical trials with large cohorts of patients being required.

Keywords: Cerebral venous thrombosis, etiology, diagnosis, treatment.

\author{
Submitted : March 16, 2021 \\ Published : April 06, 2021 \\ ISSN: 2593-8339 \\ DOI: 10.24018 /ejmed.2021.3.2.772 \\ T. G. Schreiner* \\ Clinical Rehabilitation Hospital, Iasi, \\ Romania. \\ (e-mail: \\ schreiner.thomasgabriel@yahoo.com)
}

*Corresponding Author

\section{INTRODUCTION}

From the group of cerebrovascular pathologies, cerebral venous thrombosis (CVT) remains a rare entity, having a low incidence in the general population and accounting for 0.5 $1 \%$ of all strokes [1]. Unlike ischemic stroke, which occurs mainly in people over 65 years of age, with classic cardiovascular factors (high blood pressure, dyslipidemia, type 2 diabetes, smoking, ethanol consumption, atrial fibrillation), CVT is the prerogative of young people, including children, with a peak incidence for the age range 40-55 years [2]. Moreover, women are more likely to suffer a CVT (ratio of women:men = 3:1), especially during pregnancy, postpartum or those under chronic treatment with oral contraceptives or hormone replacement therapy [3].

Despite its rarity, this pathology remains a medical emergency, fast detection and treatment being essential to reduce the morbidity and mortality associated with this condition [4]. From a clinical point of view, CVT can manifest itself in various clinical pictures, the main signs and symptoms being headache (possibly accompanied by other manifestations suggestive of intracranial hypertension syndrome), seizures, focal neurological deficit, altered consciousness to encephalopathy and coma. Depending on the manner of onset and progression of the symptoms, we distinguish 3 forms of development of the CVT: acute $(<48$ hours); subacute ( $>48$ hours, $<30$ days); chronic ( $>30$ days) $[5]$.

Once diagnosed using mainly imaging techniques, anticoagulation is essential, and in case of failure, a "heroic" endovascular approach is possible, through maneuvers such as thrombolysis or thrombectomy, but with more associated risks [6]. However, it should be noted that there are still many gaps in evidence reported by the latest guidelines, so new indepth studies regarding the optimal therapeutic approach in CVT are needed.

Thus, the aim of the paper is to present in the first part the most important data published in recent years on the etiology and diagnosis of CVT, focusing on the imaging and laboratory methods for early detection of this pathology. Subsequently, the authors discuss the pharmacological and non-pharmacological treatments useful in the acute stage, future research directions that require conducting large randomized clinical trials in order to improve patient outcome being also presented. 


\section{Diagnostic StePs In CEREBRAL Venous Thrombosis}

\section{A. Varied Etiology}

Regardless of the neurological pathology, after establishing the semiology and topographic diagnosis, determining the etiology remains essential as the first step to correctly conduct the treatment. The same principle is valid in CVT, with numerous known risk factors, that can be grouped into different categories depending on the classification. According to the latest European guide, the preferred classification is in the two categories of pathologies with a predisposition to thrombosis: on one hand the transient, correctable risk factors such as contraceptive medication, pregnancy, infections, and on the other hand the permanent risk factors such as thrombophilia, antiphospholipid syndrome, or malignancies [7]. Genetic thrombophilia is a redoubtable risk factor, of great importance being the Leiden factor $\mathrm{V}$ mutation, antithrombin deficiency, $\mathrm{C} / \mathrm{S}$ protein deficiency, prothrombin gene mutation or MTHFR mutation. The two most common mutations in the MTHFR gene (C677T and A1298C) were correlated in studies with an increased prevalence of thrombosis, both in the peripheral venous system [8] and in the brain [9]. However, the recent guidelines argue that a screening for thrombophilia does not bring a benefit on mortality or relapse rate [7]. Only the patient with a family history of venous thrombosis and those without other low risk factors could benefit from screening for thrombophilia in the idea of preventing recurrent thrombotic events by anticoagulation.

In a significant percentage of adult patients, no clear risk factor is identified. Moreover, other cardiovascular risk factors such as poorly controlled hypertension or dyslipidemia would rather suggest an ischemic arterial vascular event than a CVT. The guidelines deal particularly with the link between occult malignancies and CVT, but no direct implication between the two entities has been found and thus, screening for tumor pathology does not bring a significant benefit for the patient prognostic [10].

\section{B. The Importance of Imaging Techniques in Diagnosis}

Fast and correct diagnosis is essential in decreasing mortality in CVT patients, as seen in developed countries where the mortality rate after CVT is below $6 \%$ at 1 year [11]. Imaging investigations are the main help for the neurologist in order to obtain the correct diagnosis. This will allow the clinician to start the treatment as soon as possible, in order to offer the patient, the best chances for a complete recovery. In attempt to improve the accuracy and precocity of diagnosis, the guides compared various neuroradiological methods useful in detecting thrombi in the cerebral intravenous level. Although digital subtraction angiography (DSA) is still the gold standard, MRI venography and CT venography can be used as reliable alternatives, as there are no differences in sensitivity between Magnetic Resonance Angiography (MRA) and Computed Tomography Angiography (CTA) [12]. However, the few existing studies on this topic make the recommendations have a low level of evidence.

\section{Other Useful Laboratory Investigations}

Along with imaging investigations, laboratory tests are an essential part of supporting or refuting a diagnosis. It is the case of the detection of D-dimers, a practice often used especially when suspecting a venous thrombosis located in the lower limbs [13], this measurement bringing benefits also in the case of a CVT. According to recent studies, it is recommended to measure the level of D-dimers before neuroimaging examination in patients with suspected CVT [14], a high level of D-dimers being an additional argument for performing costly imaging examination such as MRI venography.

\section{THERAPEUTIC APPROACH OF CEREBRAL VENOUS THROMBOSIS}

\section{A. The role of Anticoagulation}

CVT treatment involves at least three distinct components, the central element being anticoagulation therapy. In adults, the medium-level recommendations according to the most recent guidelines is anticoagulation with low molecular weight heparin (LMWH) in therapeutic dose. In the acute phase, LMWH is preferred to oral anticoagulants and unfractionated heparin, in the absence of contraindications for LMWH [15]. Moreover, the presence of intracerebral hemorrhage in the initial phase is not a contraindication for anticoagulation.

The duration of anticoagulation is another intensely discussed point, the duration of chronic anticoagulant treatment varying depending on the patient. The American guide recommends chronic anticoagulation of 3-6 months for induced CVT, 6-12 months for unprovoked CVT and indefinite anticoagulation in case of recurrent CVT, thromboembolism in patient with CVT history and association of severe hemophilia [16].

However, there are still elements of uncertainty, the absence of randomized clinical trials causing gaps in the guidelines. For example, no recommendations can be made for anticoagulation in children with CVT. Also, regarding adults, there are insufficient data on the comparison between the benefits of anticoagulation therapy and other therapeutic methods such as thrombolysis or thrombectomy, or data attesting to the superiority of endovascular treatment [17].

\section{B. Symptomatic and Invasive Treatment}

The second therapeutic point of interest is represented by the symptomatic treatment that considers the management of increased intracranial pressure and epileptic seizures. The management of ICP consists of both pharmacological and invasive methods, aiming to improve symptoms and causing the regression of edema. However, the IPC syndrome found quite often in CVT does not respond satisfactorily to these methods. Evidence from clinical trials shows that acetazolamide, an inhibitor of carbonic anhydrase intensively used in neurosurgery, does not prevent death and does not improve the functional prognosis in patients with CVT [18]. Still, this medication could be used in cases of severe headache or in patients with vision problems due to compressive edema. Corticosteroids have not proven their benefits either, but they can improve the inflammatory status of some pathologies (systemic inflammatory diseases) that cause CVT [19].

Among the invasive maneuvers, lumbar puncture for therapeutic purposes can bring benefits in case of CVT- 
associated intracranial hypertension [12]. However, current guidelines do not make a clear recommendation, with more studies needed in the future in order to have evidence-based conclusions. Shunting, another neurosurgical technique used successfully in hydrocephalus of various etiologies, is not recommended for routine use in CVT-associated hydrocephalus. In addition, few studies conducted on this topic make it impossible to sustain a direct relationship between shunting and improving the morbidity and mortality index [20]. Finally, the only direction accepted in the guidelines is related to decompression surgery: although of a low level of evidence, the recommendation is to perform surgical decompression in order to prevent herniation of the brain substance and prevent death [12].

Regarding the prophylaxis of epileptic seizures, antiepileptic medication will be used in the acute phase of CVT, in patients with supratentorial lesions who have overt seizures to reduce their recurrence [21]. The onset of seizures is a common sign in CVT that increases mortality, thus, good control of epileptic activity is essential for the patient. The guide does not make recommendations for the treatment of seizures that occur during the late stage of CVT, mainly due to the side effects of chronic treatment with antiepileptic medication.

\section{Special Considerations during Pregnancy}

Mentioned in detail in both the European and the American Guidelines, the third therapeutic direction refers to the relationship between pregnancy, contraception, CVT, and the treatment of other underlying pathologies that may possibly provoke CVT. Pregnancy is an individual risk factor for CVT, however the occurrence of thrombosis during pregnancy is not a contraindication for subsequent pregnancies. The guide has clear recommendations regarding the informed consent the pregnant woman receives from the doctor about the relative and absolute risks of thrombotic events and abortion. Moreover, in women of childbearing potential, clinicians have to inform about the risks of using chronic contraceptive medication [7].

Treatment of CVT in pregnancy is represented by LMWH administered subcutaneously in therapeutic doses. In addition, in the case of pregnant women with a history of CVT, prophylaxis with LMWH is recommended if there are no other contraindications. Regarding the other pathologies that predispose to the appearance of CVT, antibiotic treatment in infections or chemotherapy in certain tumors decreases the risk of thromboembolic events [22].

\section{Future Prospects}

Although current guidelines offer satisfactory recommendations regarding the diagnosis and the possible therapeutic approaches in CVT, one can easily detect gaps of evidence, with future extensive studies being needed. Among the most important topics uncovered in the current available guidelines, we mention scarce data about the invasive approaches in CVT (thrombolysis and thrombectomy), management of CVT in children, and also potential biomarkers that may help a more rapid and high sensitivity diagnosis. Given the vast and heterogeneous nature of this future research on the topic, this article will only shortly present some recent relevant data on the invasive treatment.

Used separately and more recently combined, thrombolysis and mechanical endovascular thrombectomy are two potent tools that can be used in a CVT patient in whom anticoagulation therapy has failed. Although there are still no randomized clinical trials conducted on large groups of patients, during the recent year there are more and more single case studies in which these two invasive therapies combined have been successful. An example of innovation in the field is the therapeutic approach of (Sundar et al, 2020), who successfully performs a thrombectomy in a CVT patient whose general condition worsens after hospitalization, by using a React 71 (large size) device [23]. Similar endovascular approaches with favorable short- and long-term outcomes are found in the recent literature, also in much younger patients (a 5-year-old child and a 10-day-old infant) [24], [25].

Given the rarity of CVT, retrospective studies conducted over several years are one of the safest solutions to provide a statistically significant large group of patients and to offer the possibility to test and compare invasive procedures with the anticoagulant treatment currently used. One of the few retrospective cohort studies conducted until present showed that stent retriever thrombectomy combined with long-term local thrombolysis (SRT-LLT) is a safe procedure for patients with severe hemorrhagic CVT, opening new perspectives for more invasive treatment approaches [26].

According to literature, invasive methods are often used off-label in the clinical setting, with some impressive singular cases in which different devices (large bore intermediate suction, stent retriever thrombectomy) have been successful [27]. However, it is clear the need for large new cohort studies in both adults and children, focusing on cases in which anticoagulation and symptomatic treatment are not sufficient.

\section{CONCLUSION}

Although rare, CVT is a potentially lethal disease that affects young and professionally active people, especially women, including pregnant women. Despite a diverse initial clinical picture, the neurologist must conduct a quick clinical examination and make a rapid diagnosis, with imagery techniques such as CTA and MRA both of great importance in sustaining the diagnostic. Anticoagulation with LMWH is the main therapeutic resource, to which is added the symptomatic therapy aimed to reduce edema and the risk of epileptic seizures. Current guidelines provide satisfactory diagnostic and therapeutic recommendations, however, there are some future directions of research to be followed. New studies are needed in order to impose guideline directions regarding the approach and management of CVT in children. Randomized clinical trials on thrombolysis and thrombectomy should be performed, focusing on both young and old CVT patients. Finally, new methods of diagnosis and treatment must be found in order to reduce the mortality of the CVT patient.

\section{ACKNOWLEDGMENT}

The author declares no conflict of interest. There was no funding applied to this article. The data supporting the findings of this study are available within the article. 


\section{REFERENCES}

[1] Danwang C, Mazou TN, Tochie JN, et al. Global epidemiology and patterns of cerebral venous thrombosis: a systematic review and metaanalysis protocol. BMJ Open 2018;8:e019939.

[2] Capecchi M, Abbattista M, Martinelli I. Cerebral venous sinus thrombosis. J Thromb Haemost 2018; 16: 1918-31.

[3] Ibrahim N, El-Shahawy A, Elshabacy A. Risk of cerebral venous thrombosis in oral contraceptives pills users. The Egyptian Journal of Radiology and Nuclear Medicine. 2018; 49 10.1016/j.ejrnm.2018.02.002.

[4] Stack CA \& Cole JW (November 5th, 2018). Cerebral Venous Thrombosis: A Clinical Overview, Ischemic Stroke of Brain, Pratap Sanchetee, IntechOpen, DOI: 10.5772/intechopen.79049. Available from: https://www.intechopen.com/books/ischemic-stroke-ofbrain/cerebral-venous-thrombosis-a-clinical-overview.

[5] Tadi P, Behgam B, Baruffi S. Cerebral Venous Thrombosis. [Updated 2020 Aug 10]. In: StatPearls [Internet]. Treasure Island (FL): StatPearls Publishing; 2020 Jan-. Available from: https://www.ncbi.nlm.nih.gov/books/NBK459315/.

[6] Lee S, Mokin M, Hetts SW on behalf of the Society of NeuroInterventional Surgery, et al. Current endovascular strategies for cerebral venous thrombosis: report of the SNIS Standards and Guidelines Committee. Journal of NeuroInterventional Surgery 2018;10:803-810.

[7] Ferro JM, Bousser MG, Canhão P, Coutinho JM, Crassard I, Dentali F, di Minno M, Maino A, Martinelli I, Masuhr F, Aguiar de Sousa D, Stam J; European Stroke Organization. European Stroke Organization guideline for the diagnosis and treatment of cerebral venous thrombosis - endorsed by the European Academy of Neurology. Eur J Neurol. 2017 Oct;24(10):1203-1213.

[8] Gogu AE, Jianu DC, Dumitrascu V, Ples H, Stroe AZ, Docu Axelerad D, Docu Axelerad A. MTHFR Gene Polymorphisms and Cardiovascular Risk Factors, Clinical-Imagistic Features and Outcome in Cerebral Venous Sinus Thrombosis. Brain Sci. 2020 Dec 27;11(1):E23.

[9] Bostan A, Tapoi LC, Barcan MN, Florea L. Cerebral vein thrombosis associated with MTHFR A1289C mutation gene in a young postpartum woman. Arch Clin Cases 2019; 6(1):26-30.

[10] Carrier M, Lazo-Langner A, Shivakumar S, et al. SOME Investigators. Screening for occult cancer in unprovoked venous thromboembolism. N Engl J Med 2015; 20:697-704.

[11] Kristoffersen ES, Harper CE, Vetvik KG, Zarnovicky S, Hansen JM, Faiz KW. Incidence and Mortality of Cerebral Venous Thrombosis in a Norwegian Population. Stroke. 2020 Oct;51(10):3023-3029.

[12] Ferro JM, Aguiar de Sousa D. Cerebral Venous Thrombosis: An Update. Curr Neurol Neurosci Rep. 2019 Aug 23;19(10):74.

[13] Li J, Zhang F, Liang C, Ye Z, Chen S, Cheng J. The Diagnostic Efficacy of Age-Adjusted D-Dimer Cutoff Value and Pretest Probability Scores for Deep Venous Thrombosis. Clin Appl Thromb Hemost. 2019;25:1076029619826317.

[14] Heldner MR, Zuurbier SM, Li B, Von Martial R, Meijers JCM, Zimmermann R, Volbers B, Jung S, El-Koussy M, Fischer U, Kohler HP, Schroeder V, Coutinho JM, Arnold M. Prediction of cerebral venous thrombosis with a new clinical score and D-dimer levels. Neurology. 2020 Aug 18;95(7):e898-e909.

[15] Qureshi A, Perera A. Low molecular weight heparin versus unfractionated heparin in the management of cerebral venous thrombosis: A systematic review and meta-analysis. Ann Med Surg (Lond). 2017;17:22-26.

[16] Field TS, Hill MD. Cerebral Venous Thrombosis. Stroke. 2019 Jun;50(6):1598-1604. doi: 10.1161/STROKEAHA.119.025334. Epub 2019 May 16. PMID: 31092159.

[17] Coutinho JM, Zuurbier SM, Bousser MG, Ji X, Canhão P, Roos YB, Crassard I, Nunes AP, Uyttenboogaart M, Chen J, Emmer BJ, Roosendaal SD, Houdart E, Reekers JA, van den Berg R, de Haan RJ, Majoie CB, Ferro JM, Stam J; TO-ACT investigators. Effect of Endovascular Treatment with Medical Management vs Standard Care on Severe Cerebral Venous Thrombosis: The TO-ACT Randomized Clinical Trial. JAMA Neurol. 2020 Aug 1;77(8):966-973.

[18] Beiruti K, Shadi J, Shahien R, Keigler G. Critical management of cerebral venous sinus thrombosis (CVST) concomitant with high intracranial pressure (ICP). J Neurol Sci 2017; 1103: 381, SUPPLEMENT 398.

[19] Sorgun MH, Rzayev S, Kural MA, Erdoğan S, Yücesan C. Cerebral Venous Thrombosis in Behçet's Disease Patients Compared to Other Causes of Cerebral Venous Thrombosis: A Retrospective Study. Arch Rheumatol. 2016 May 17;31(3):248-253.
[20] Lobo S, Ferro JM, Barinagarrementeria F, Bousser MG, Canhão P Stam J; ISCVT Investigators. Shunting in acute cerebral venous thrombosis: a systematic review. Cerebrovasc Dis. 2014;37(1):38-42.

[21] Mehvari Habibabadi J, Saadatnia M, Tabrizi N. Seizure in cerebral venous and sinus thrombosis. Epilepsia Open. 2018;3(3):316-322.

[22] Stam JM, Bousser MG, Canhão P, Coutinho JM, Crassard I, Dentali F, et al. European Stroke Organization guideline for the diagnosis and treatment of cerebral venous thrombosis: endorsed by the European Academy of Neurology. Eur J Neurol. 2017; 24(10): 1203-13.

[23] Sundar K, Paulraj S, Choudhury SR, Hassan H, Sengupta J, Pattari SK Successful Endovascular Treatment of Cerebral Venous Thrombosis with a Novel, Larger Aspiration Catheter (REACT): A Case Report. Neurointervention. 2020 Dec 28. doi: 10.5469/neuroint.2020.00304. Epub ahead of print. PMID: 33355855.

[24] Cellerini M, Francavilla R, Testoni C, Maffei M, Zucchelli M, Ghizzi C. Dural sinus mechanical thrombectomy and continuous local rt-PA infusion in a child with refractory intracranial hypertension and progressive visual loss: A case report. Surg Neurol Int. 2020 Aug 21;11:253.

[25] Zelenak K, Uhrikova Z, Mikler J, Zibolen M. Mechanical Thrombectomy for Cerebral Venous Sinus Thrombosis in a Neonate. Indian Pediatr. 2020 Sep 15;57(9):862-863. PMID: 32999120.

[26] Wang Y, Zhao C, Huang D, Sun B, Wang Z. Stent retriever thrombectomy combined with long-term local thrombolysis for severe hemorrhagic cerebral venous sinus thrombosis. Exp Ther Med. 2020 Nov;20(5):66

[27] Lewis W, Saber H, Sadeghi M, Rajah G, Narayanan S. Transvenous Endovascular Recanalization for Cerebral Venous Thrombosis: A Systematic Review and Meta-Analysis. World Neurosurg. 2019 Oct;130:341-350.

Thomas Gabriel Schreiner, born on 27.12.1993 in Iasi, Romania, is a medical doctor (M.D.), currently working as a neurologist in the Neurology Department of the Clinical Rehabilitation Hospital in Iasi. He has graduated the University of Medicine and Pharmacy "Gr. T. Popa" Iasi and is now a PhD student at the University of Medicine and Pharmacy "Carol Davila" of Bucharest, conducting his thesis in the field of neurodegenerative diseases, one of his main fields of interest.

$\mathrm{He}$ is also interested in multiple sclerosis, neurological rehabilitation, and cerebrovascular diseases, these being the main neurological disorders encountered in his daily clinical practice. Moreover, he showed a constant interest for clinical and fundamental research, proof being his latest works published in international medical journals during the last years.

Mr. Schreiner is also an active member of some of the most important international societies for neurology and neurosciences (EAN, IASP, RSN, IBBS), participating regularly in meetings and conferences. 